\title{
Epigenetic inheritance of an inducibly nucleosome-depleted promoter and its associated transcriptional state in the apparent absence of transcriptional activators Ryosuke Ohsawa, Melissa Adkins and Jessica K Tyler*
}

Address: Department of Biochemistry and Molecular Genetics, University of Colorado School of Medicine, Aurora, CO, USA

Email: Ryosuke Ohsawa - ryosuke.ohsawa@ucdenver.edu; Melissa Adkins - melissa.adkins@uchsc.edu; Jessica K Tyler* - jessica.tyler@uchsc.edu

* Corresponding author

Published: II September 2009

Epigenetics \& Chromatin 2009, 2:II doi:10.1186/I756-8935-2-II
Received: 22 May 2009

Accepted: II September 2009

This article is available from: http://www.epigeneticsandchromatin.com/content/2/I/II

(C) 2009 Ohsawa et al; licensee BioMed Central Ltd.

This is an Open Access article distributed under the terms of the Creative Commons Attribution License (http://creativecommons.org/licenses/by/2.0), which permits unrestricted use, distribution, and reproduction in any medium, provided the original work is properly cited.

\begin{abstract}
Background: Dynamic changes to the chromatin structure play a critical role in transcriptional regulation. This is exemplified by the Spt6-mediated histone deposition on to histone-depleted promoters that results in displacement of the general transcriptional machinery during transcriptional repression.

Results: Using the yeast PHO5 promoter as a model, we have previously shown that blocking Spt6mediated histone deposition on to the promoter leads to persistent transcription in the apparent absence of transcriptional activators in vivo. We now show that the nucleosome-depleted PHO5 promoter and its associated transcriptionally active state can be inherited through DNA replication even in the absence of transcriptional activators. Transcriptional reinitiation from the nucleosomedepleted $\mathrm{PHO} 5$ promoter in the apparent absence of activators in vivo does not require Mediator. Notably, the epigenetic inheritance of the nucleosome-depleted PHO5 promoter through DNA replication does not require ongoing transcription.
\end{abstract}

Conclusion: Our results suggest that there may be a memory or an epigenetic mark on the nucleosome-depleted $\mathrm{PHO} 5$ promoter that is independent of the transcription apparatus and maintains the promoter in a nucleosome-depleted state through DNA replication.

\section{Background}

Histone removal, also termed nucleosome disassembly, from promoter regions is a recently-discovered mechanism of transcriptional regulation that is largely conserved throughout the eukaryotes [1-11]. The function of chromatin disassembly at promoters has been revealed from studies at the yeast PHO5 promoter. Histone removal is required to allow access of the general transcription machinery to the promoter in order to initiate transcription $[1,12]$. Conversely, repression of the PHO5 promoter is accompanied by chromatin reassembly [13]. Chromatin reassembly at the promoter is mediated by the histone chaperone Spt6 and is essential for transcriptional repression because the histones effectively compete with the general transcription machinery for occupancy at the PHO5 promoter [13].

The yeast $\mathrm{PHO} 5$ gene encodes an acid phosphatase and its expression is tightly regulated by intracellular phosphate levels. In low phosphate conditions, the sequence-specific transactivator Pho4 is dephosphorylated causing it to localize to the nucleus where it binds the $\mathrm{PHO} 5$ promoter $[14,15]$. Pho4 binding to the DNA initiates depletion of the four positioned nucleosomes that normally reside 
over the PHO5 promoter including the Pho4 binding site termed UASp2 and the TATA box [16]. In repressing conditions (high phosphate), Pho4 is phosphorylated by Pho80-Pho85, which causes its export to the cytoplasm $[14,17]$. Loss of Pho4 from the promoter leads to the histones being reassembled by Spt6 at the PHO5 promoter [16] (Figure 1). We have previously shown that inactivation of Spt6 prior to addition of the signal for repression (phosphate) results in the PHO5 promoter remaining nucleosome-depleted and transcriptionally active in vivo, even though the activators no longer occupy the promoter [13]. This indicates that the main role of some transcriptional activators is to maintain promoters in a nucleosome-depleted state, which in turn indirectly allows the binding of the general transcription machinery to the core promoter.

In this work, we set out to determine whether the inducibly nucleosome-depleted PHO5 promoter and its associated transcriptionally active state can be inherited through DNA replication in the absence of the activators that originally initiated this state. We discovered that inactivation of the chromatin assembly factor Spt6 enables the PHO5 promoter to be maintained in a nucleosome-depleted state through DNA replication even when the activator that originally signaled for promoter chromatin disassembly was exported to the cytoplasm prior to DNA replication. Basically, we have achieved epigenetic inheritance of a nucleosome-depleted DNA state facilitating persistent transcription in the absence of transcriptional activators in vivo. Furthermore, we show that ongoing transcription is not required for the inheritance of this inducibly nucleosome-depleted promoter region. Our results suggest that there may be an epigenetic signal that can maintain promoters in a nucleosome-depleted state through replication.

\section{Results}

In low phosphate conditions, binding of the transcriptional activator Pho4 to the PHO5 promoter is the signal for chromatin disassembly from the promoter. In high phosphate conditions, disengagement of Pho4 is the signal for Spt6-mediated chromatin reassembly of the PHO5 promoter (Figure 1(a)). Consequently, inactivation of the histone chaperone Spt6 using isogenic strains carrying either the spt6-140 or spt6-1004 temperature-sensitive allele prevented transcriptional repression of $\mathrm{PHO} 5$ in asynchronous cultures in response to the addition of phosphate (Figure 1(b)), even though we have previously shown that phosphate addition results in the rapid loss of Pho 2 and Pho4 from the PHO5 promoter when Spt6 is inactivated [13]. This persistent $\mathrm{PHO} 5$ transcription under repressing conditions in the apparent absence of Spt 6 and activators is due to failure to reassemble chromatin on to the PHO5 promoter (Figure 1(c)) [13], which enables continued access of the general transcription machinery to the promoter.

It is generally believed that all newly replicated DNA is reassembled into chromatin following DNA replication. Therefore, we asked whether the nucleosome-depleted PHO5 promoter in the absence of activators (that is, in the spt6 mutant +Pi condition) would also be reassembled into chromatin following DNA replication. To ensure passage through DNA replication had occurred, we arrested our wild type (WT) and spt6-140 PHO5 expressing cultures (-Pi) growing at $23^{\circ} \mathrm{C}$ in $\mathrm{G}_{1}$ phase with the mating pheromone alpha factor. Following cell cycle arrest, we split the cultures into two and placed one culture at $23^{\circ} \mathrm{C}$ and the other at $39^{\circ} \mathrm{C}$ to inactivate Spt6 (Figure 2(a)). After inactivation of Spt6, we added phosphate as the signal for Pho4 eviction from the promoter and removed the alpha factor to allow re-entry into the cell cycle. Passage through S-phase was confirmed by flow cytometry analysis (Figure 2(a)). The phosphatase activity of Pho5 confirmed that the WT strain at both temperatures and the spt6 strain at $23^{\circ} \mathrm{C}$ repressed PHO5 transcription upon addition of phosphate (Figure 2(b)). By contrast, the spt6 strain at $39^{\circ} \mathrm{C}$ failed to repress $\mathrm{PHO} 5$ transcription following passage through S-phase (Figure 2(b)), as in asynchronous cultures (Figure 1(b)), even though the activators should have disengaged from the promoter in response to phosphate addition [13]. As Spt6-mediated chromatin reassembly is absolutely required for $\mathrm{PHO} 5$ repression [13], the failure to repress PHO5 following DNA replication in the absence of functional Spt6 (Figure 2(b)) indicated that the chromatin may not be reassembled over the PHO5 promoter following DNA replication in spt6 mutants. We confirmed this prediction by chromatin immunoprecipitation (ChIP) analysis against the Cterminus of histone $\mathrm{H} 3$ using primer pairs spanning the TATA box, the UASp2 Pho4 binding site and the region adjacent to the PHO5 promoter (Figure 2(c)). This analysis showed that the chromatin fails to be reassembled on to the PHO5 promoter following DNA replication in the absence of Spt6, even though the activators (the signal to maintain chromatin disassembly) are no longer bound to the promoter (Figure 2(d)-2(g)) (13). Importantly, our results indicate that $\mathrm{Spt} 6$ does not mediate global replication-dependent chromatin assembly because the region adjacent to the nucleosome-depleted PHO5 promoter was reassembled into chromatin following DNA replication even when Spt6 was inactivated (Figure 2(h) and 2(i)). These results suggest that there is some factor or epigenetic mark at the PHO5 promoter that is signaling for it to remain nucleosome-depleted through DNA replication even in the absence of the transcriptional activators that initially triggered the nucleosome depletion.

Next, we set out to identify the nature of the epigenetic mark or factor on the $\mathrm{PHO} 5$ promoter that signals for it to 

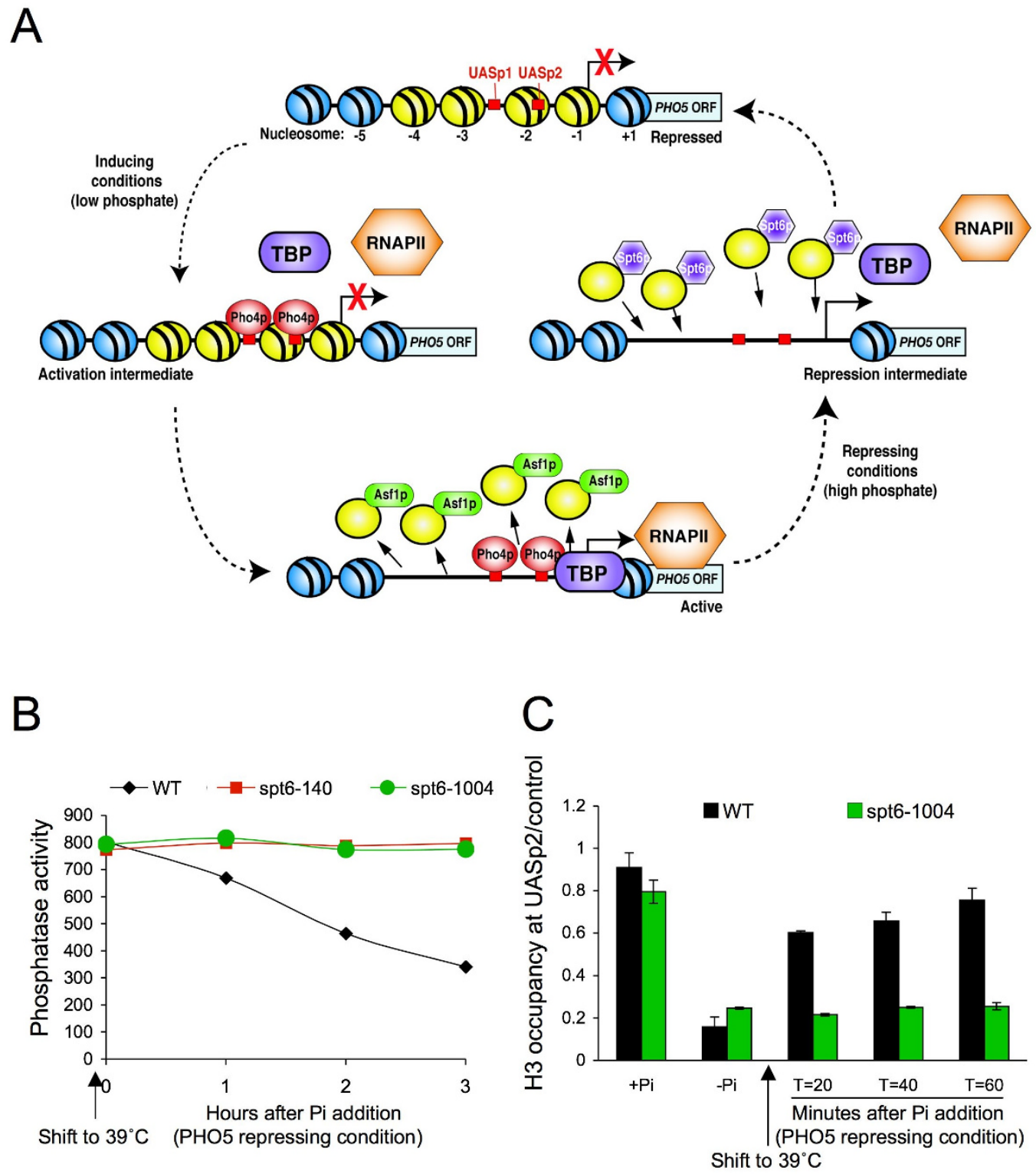

Figure I

Spt6-mediated chromatin assembly and transcriptional repression at the PHO5 promoter. (a) UASpI and UASp2 are binding sites for the Pho2 and Pho4 transactivators. During activation, chromatin disassembly of the four yellow nucleosomes is promoted by Asfl to allow access of the general transcription machinery to the promoter. During repression, chromatin is reassembled over the $\mathrm{PHO} 5$ promoter by the histone $\mathrm{H} 3 / \mathrm{H} 4$ chaperone Spt6 to compete with the general transcription machinery for DNA binding. (b) Strain JKT00I0 (WT), JMY0002 (spt6-l 40), and MAY0067 (spt6-1004) were grown in phosphate-depleted media to activate $\mathrm{PHO} 5$ transcription. Following a 4 hour shift to $39^{\circ} \mathrm{C}$ to inactivate Spt6, phosphate was added as a signal for $\mathrm{PHO} 5$ repression. Samples were assayed for phosphatase activity at the indicated times after addition of phosphate. (c) Strain JKT00I0 (WT) and MAY0067 (spt6-1004) were initially grown in phosphate-rich media (+Pi) where $\mathrm{PHO} 5$ is repressed, then shifted to phosphate-depleted media to activate $\mathrm{PHO5}$ transcription (-Pi). Following a 2 hour shift to $39^{\circ} \mathrm{C}$, phosphate was added. Samples were taken at the indicated times, and analyzed for histone occupancy at $\mathrm{PHO} 5$ UASp2 by chromatin immunoprecipitation (ChIP) analysis. The amount of immunoprecipitated DNA was determined by quantitative PCR. As a control, primer sets were used for TELVIR. Quantitation of H3 levels over the UASp2 region is a ratio of immunoprecipitated UASp2 product relative to the immunoprecipitated TELVIR product divided by the ratio of input UASp2 product relative to the ratio of input TELVIR product. Averages of three independent experiments are shown; error bars indicate the $95 \%$ confidence interval. 
A

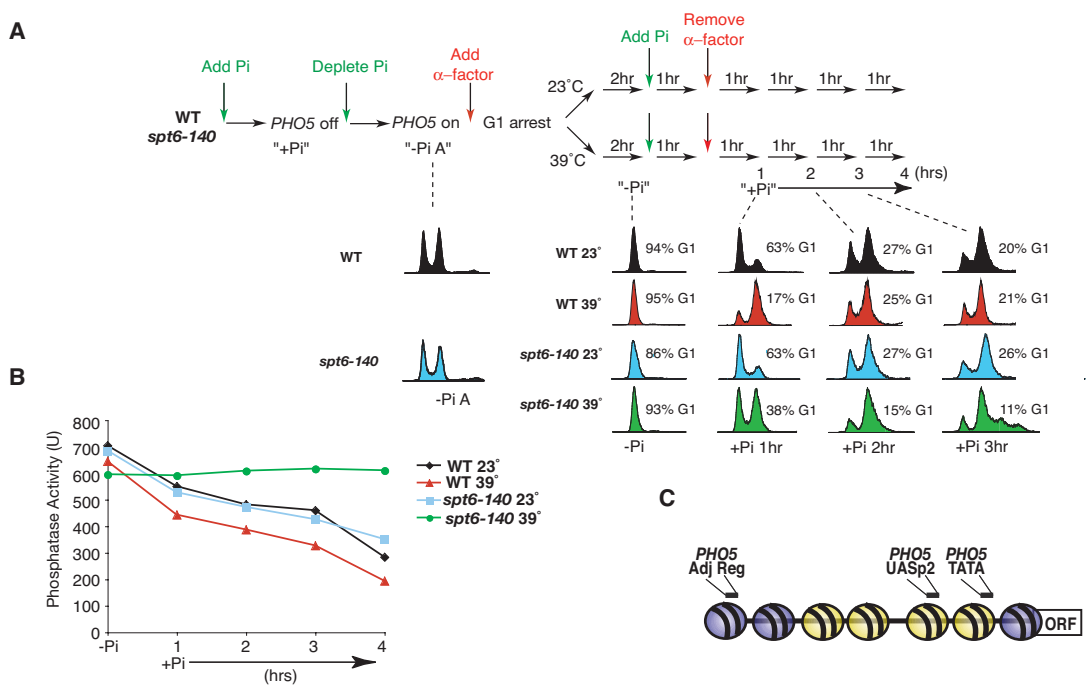

D

B
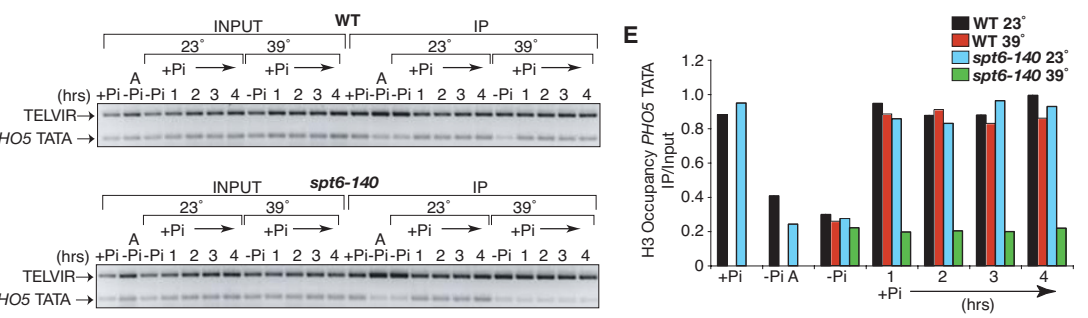

$\mathbf{F}$
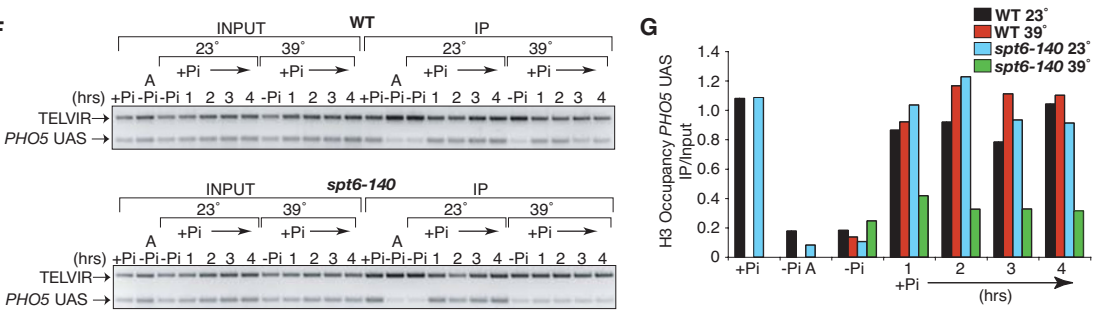

H
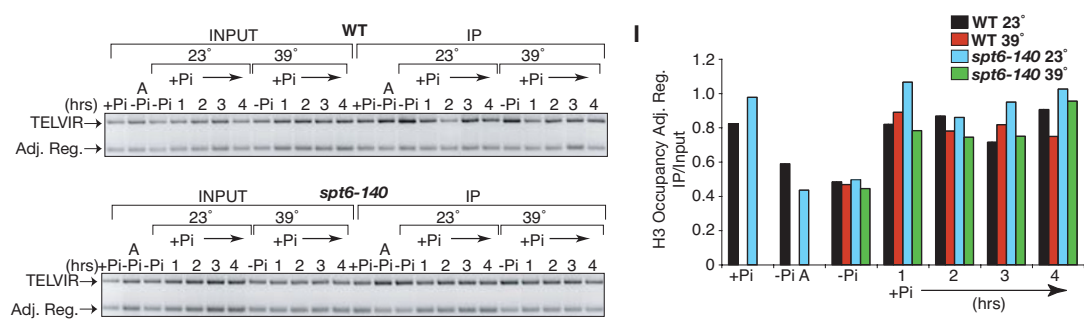

Figure 2

The naked $\mathrm{PHO} 5$ promoter can be inherited through DNA replication even in the absence of the activators. (a) Schematic of experimental outline, showing flow cytometry DNA content profiles for the strains JKT00I0 (WT) and JMY0002 (spt6-140) at the indicated temperatures. '-Pi A' refers to asynchronously growing in phosphate-free media, while the remainder of the $-\mathrm{Pi}$ samples are at various stages of the cell cycle, as indicated by the flow cytometry analyses below. (b) Inactivation of Spt6 prevents repression of $\mathrm{PHO} 5$ even following DNA replication. Acid phosphatase activity of the PHO5 gene product was measured at the indicated times in the schematic shown in (a). (c) Schematic of locations of primer pairs used for the chromatin immunoprecipitation (ChIP) analyses shown below. (d) ChIP analysis of H3 occupancy at the PHO5 TATA region. (e) Quantitation of ChIP analysis of $\mathrm{H} 3$ occupancy at the PHO5 TATA region, normalized to the TELVIR internal control. (f) ChIP analysis of $\mathrm{H} 3$ occupancy at the PHO5 UAS region. (g) Quantitation of ChIP analysis of $\mathrm{H} 3$ occupancy at the PHO5 UAS region, normalized to the TELVIR internal control. (h) ChIP analysis of $\mathrm{H} 3$ occupancy at the PHO5 adjacent region. (i) Quantitation of ChIP analysis of $\mathrm{H} 3$ occupancy at the PHO5 adjacent region, normalized to the TELVIR internal control. 
remain nucleosome-depleted even after DNA replication in the apparent absence of activators. Given that the histones are mostly depleted from the promoter, we focused on the transcription machinery rather than chromatin modifications or chromatin remodelers per se. First, we asked whether Mediator is required for continued transcription from the nucleosome-depleted promoter in the absence of activators, because Mediator is known to be required for transcriptional reinitiation in vitro $[18,19]$. To inactivate Mediator, we used a conditional mutant of the Mediator subunit Srb4. We first confirmed that Mediator is required for transcriptional initiation from the PHO5 promoter in vivo. As inactivation of Srb 4 by a shift to $39^{\circ} \mathrm{C}$ blocks cell growth, we performed these experiments in the context of a temperature-sensitive mutation of $\mathrm{PHO} 80$, which allowed us to bypass the need for cell growth (in order to use up cellular phosphate stores) that is normally required for PHO5 induction. In this experiment, we clearly see that $\mathrm{PHO} 5$ is induced upon inactivation of Pho80 as expected, and that this induction of PHO5 requires Srb4 because it does not occur in the pho80 srb4 double mutant (Figure 3(a)). As such, Mediator is required for initiation of transcription from the PHO5 gene.

Next, we asked whether Mediator is required for the persistent transcription that occurs from the nucleosomedepleted PHO5 promoter in the absence of activators. Given that the nucleosome-depleted promoter can be maintained through DNA replication (Figure 2), all subsequent repression experiments were performed in asynchronously cycling cells. We shifted the PHO5 expressing cultures to $39^{\circ} \mathrm{C}$ to inactivate both Mediator and Spt6, followed by addition of the repressive signal (phosphate). Notably, we observed a repression of transcription that was due to the heat shock alone, as it was seen even before we added the phosphate to repress PHO5 transcription (Figure 3(b), at $T=0$ hour). The reason why the heat shock alone partially represses PHO5 transcription is unclear, but it is accompanied by partial reassembly of the chromatin in an Spt6-independent manner upon the heat shock (see later). Notwithstanding, the completion of promoter chromatin assembly and the full transcriptional repression that is mediated by Spt6 were still apparent after adding phosphate to the WT and spt6 strain (Figure $3(\mathrm{~b})$ ). Interestingly, we reproducibly find that inactivation of Srb4 does not prevent the persistent transcription that occurs in the absence of Spt6 (Figure 3(b)). This result indicates that Mediator is not required for transcriptional reinitiation of activator-independent transcription from a nucleosome-depleted promoter in vivo. This is in contrast to the requirement for Mediator in transcriptional reinitiation of activator-independent transcription from naked DNA templates in vitro $[18,19]$, suggesting that the in vitro transcription systems may not fully recapitulate the situation in vivo.

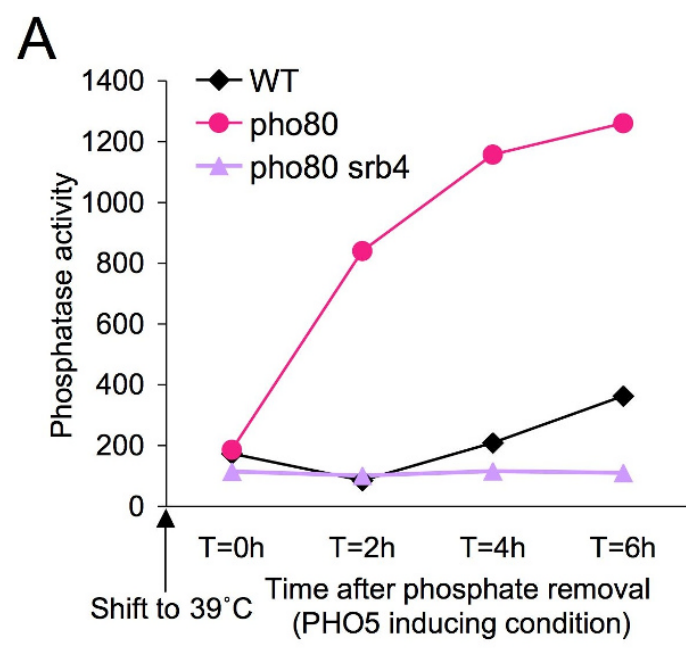

B

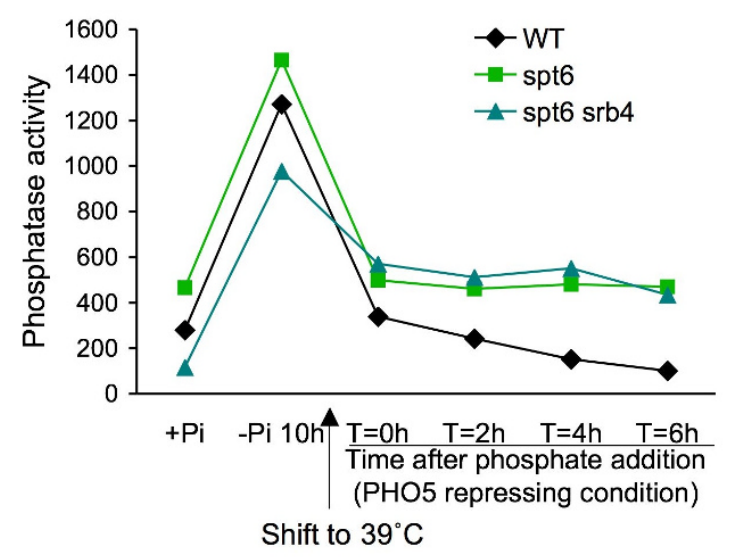

Figure 3

Mediator is not required for $\mathrm{PHO} 5$ transcription in the absence of activators in vivo. (a) Mediator is required for transcriptional initiation of $\mathrm{PHO} 5$ in vivo. Strains JKT00IO (WT), ROY0IO (pho80), and ROY0I I (pho80 srb4) growing in phosphate containing media (PHO5 repressed) were shifted to $39^{\circ} \mathrm{C}$ in low phosphate media, followed by analysis of $\mathrm{PHO} 5$ induction via the phosphatase activity assay. (b) Mediator is not required for continued transcription from the nucleosome-depleted promoter in the absence of activators. Strains JKT0010 (WT), JMY002 (spt6), and SKW067 (spt6 srb4) were shifted to $39^{\circ} \mathrm{C}$ while PHO5 transcription was occurring in low phosphate media, followed by the addition of phosphate as the signal to repress $\mathrm{PHO} 5$ transcription and analysis of $\mathrm{PHO} 5$ induction via the phosphatase activity assay.

Next, we asked whether transcription itself was required for the maintenance of the nucleosome-depleted promoter through DNA replication in the absence of transcriptional activators in vivo. To prevent PHO5 transcription, we used a strain with a point mutation in the PHO5 TATA box [20] (Figure 4(a))). As expected, mutation of the PHO5 TATA box prevented transcription 
from PHO5 (Figure 4(b)). Next, we examined whether mutation of the PHO5 TATA box influenced the failure of chromatin to be reassembled onto the promoter following DNA replication in the spt6 mutant upon addition of phosphate (the signal for repression). We found that the promoters of the TATA box mutants were not disassembled quite as well as the normal TATA box strains (Figure 4(c) and 4(d)). Upon addition of phosphate as the signal for repression, the extent of chromatin reassembly in the spt6 mutants with and without the TATA box were equally impaired as compared with the efficient chromatin reassembly observed in the WT and the WT TATA mutant at both the TATA box and the UASp2 site (Figure 4(c) and 4(d)). These results indicate that transcription from the PHO5 promoter is not required to maintain the promoter in a nucleosome-depleted state through DNA replication.

\section{Discussion \\ Epigenetic inheritance of an inducibly nucleosome- depleted promoter in the apparent absence of transcriptional activators}

We have developed a system that enables epigenetic inheritance of expression of the PHO5 gene in the apparent absence of transcriptional activators by maintaining the promoter in a nucleosome-depleted state. We achieved this situation by inactivating the Spt 6 chromatin assembly factor prior to addition of the repression signal (phosphate) for PHO5 transcription. The phosphate signals for the activators to dissociate from the $\mathrm{PHO} 5$ promoter, while inactivation of Spt6 prevents reassembly of the PHO5 promoter into chromatin. Surprisingly, we found that this naked $\mathrm{PHO} 5$ promoter remains naked through DNA replication, even though the original signal for chromatin disassembly, the activators, left the promoter prior to DNA replication. This epigenetic inheritance of the nucleosome-depleted promoter does not require the Pho2 and Pho4 activators, Mediator, nor transcription per se, suggesting that an epigenetic mark is retained on the promoter to maintain the nucleosomedepleted state through DNA replication.

PHO5 transcription normally requires high levels of unphosphorylated Pho4 and Pho2 to be bound to the PHO5 promoter. The fact that PHO5 repression occurs when the Pho4 and Pho2 activators leave the promoter indicates that they do not function via a 'hit-and-run' mechanism. Furthermore, all the evidence indicates that Pho 4 and Pho 2 no longer occupy the PHO5 promoter during the epigenetic inheritance of the nucleosomedepleted promoter in the spt6 mutant. Upon phosphate addition, the Pho80-Pho85 cyclin-cyclin-dependent kinase complex phosphorylates Pho4, resulting in seemingly complete nuclear export of Pho4 by 3 to 6 minutes after phosphate addition [21]. Even when phosphorylated Pho4 is made to remain in the nucleus it does not activate
PHO5 transcription [22]. This is because phosphorylated Pho4 fails to bind to Pho2, and the interaction between Pho 4 and Pho2 is required for recruitment of Pho4 to the PHO5 promoter [23]. Our previous studies in the same spt6 mutant strain and same growth conditions that we used in this work demonstrated that 1 hour after adding phosphate to the media Pho2 and Pho4 no longer occupied the PHO5 promoter (by ChIP analysis and in vivo dimethyl sulfate footprinting analyses) and Pho4 had left the nucleus (by Pho4 green fluorescent protein localization analysis) [13]. It should be noted that even without synchronization of the cells prior to DNA replication with alpha factor, the extended period of growth in phosphatedepleted media that is required to activate PHO5 transcription leads to cell synchronization prior to DNA replication. This is because after the cells have used up their polyphosphate stores they are unable to obtain any more phosphate to make nucleotides, resulting in arrest prior to DNA replication. Conversely, replication resumes upon addition of phosphate, which is the stimulus for PHO5 repression, because nucleotide synthesis resumes. As such, the continued $\mathrm{PHO} 5$ transcription that we observed previously in the absence of activators in the spt6 mutant without alpha factor arrest of the cells reflected the maintenance of the nucleosome-depleted and transcriptionally active state of the PHO5 promoter through DNA replication [13].

It has always been assumed that the entire genome is rapidly reassembled into chromatin following every round of DNA replication. The majority of persistently nucleosome-free regions in the genome are thought to be nucleosome-free due to their AT-rich sequences, which are rigid and therefore incorporated poorly into nucleosomes [2427]. By contrast, nucleosomes are depleted from promoters in response to transcriptional activator binding. Following DNA replication, transcriptional activators presumably rebind to their sites and signal for the disassembly of chromatin from promoters again. However, in our system the PHO5 promoter remains nucleosomedepleted through DNA replication even in the absence of activators (Figure 2). As our system requires that Spt6 be inactivated, it was essential to show that Spt6 is not mediating global replication-dependent chromatin assembly, which we did by demonstrating that the region adjacent to the PHO5 promoter reassembles into chromatin following DNA replication in the absence of functional Spt6 (Figure 2(h) and 2(i)). To our knowledge, this is the first example of a system for studying the epigenetic inheritance of an inducibly nucleosome-depleted DNA state. Mechanistically, we do not know if the histones are reassembled on to the PHO5 promoter by the global replication-dependent chromatin reassembly apparatus and then rapidly disassembled after replication, or whether the PHO5 promoter was never reassembled into chroma- 
A

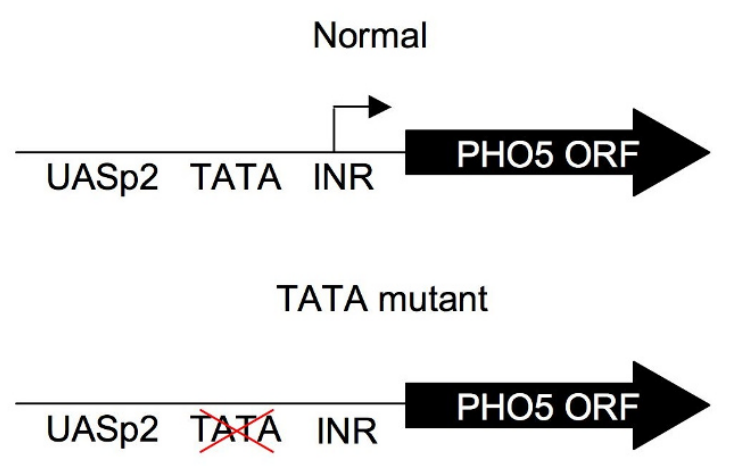

C

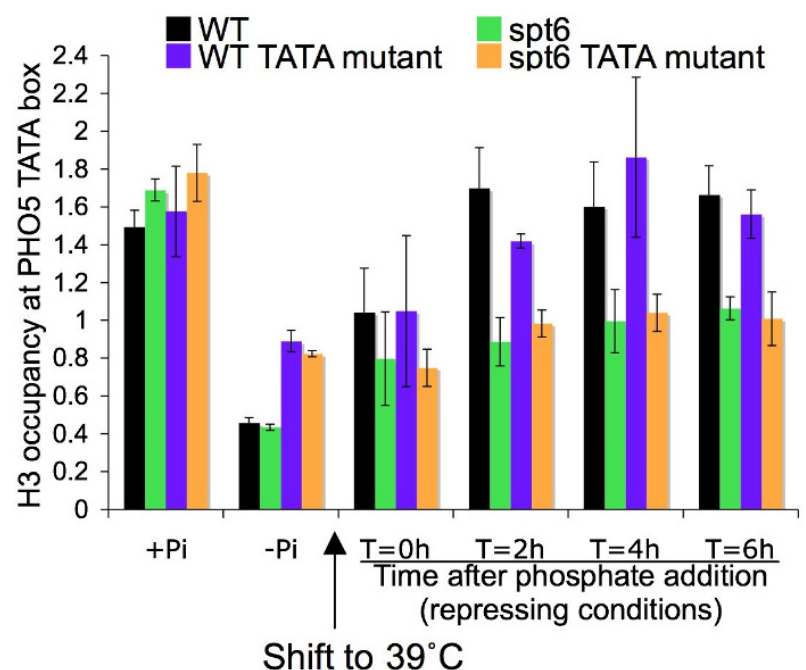

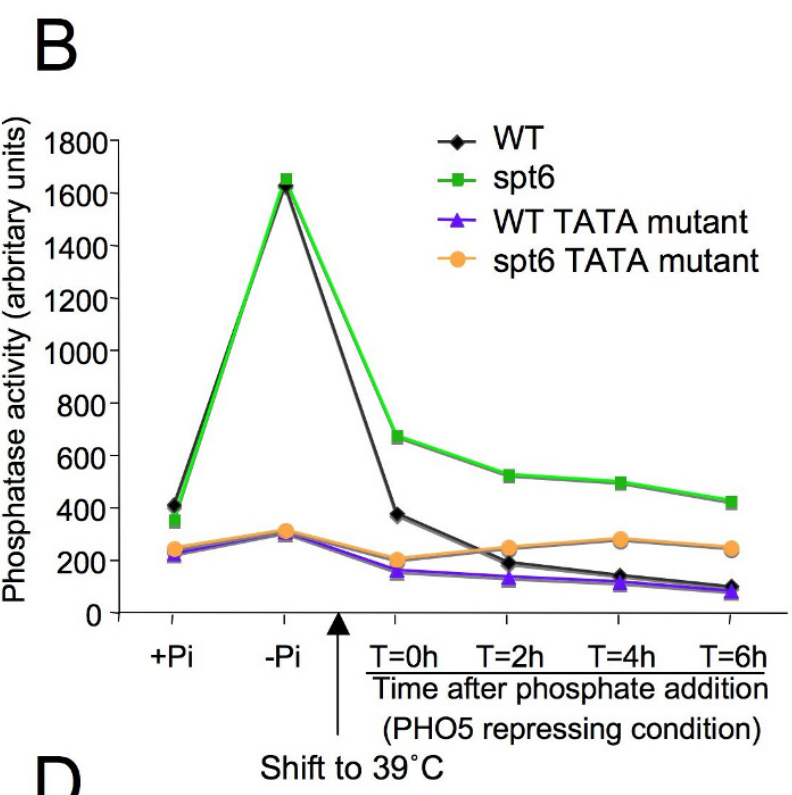

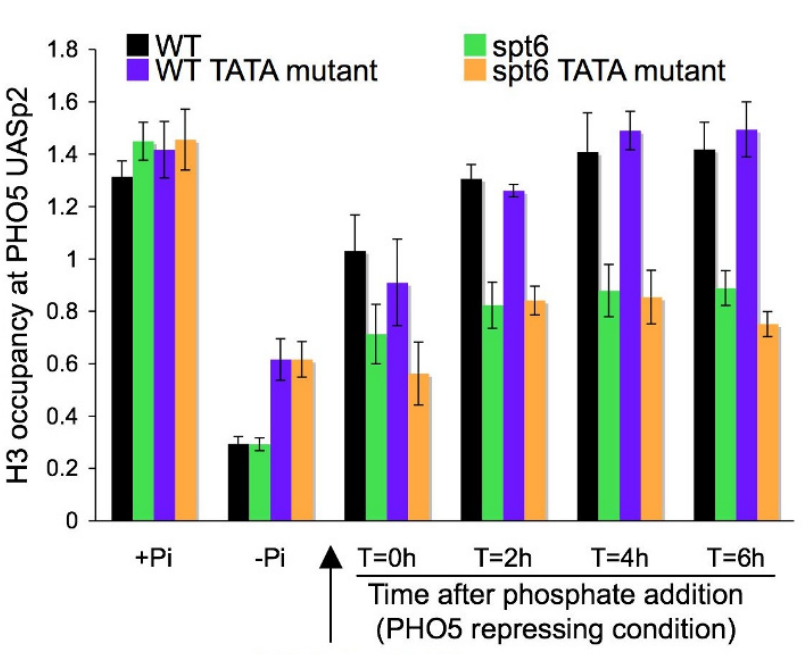

Shift to $39^{\circ} \mathrm{C}$

\section{Figure 4}

Maintenance of the nucleosome-depleted PHO5 promoter does not require persistent transcription. (a) Schematic of constructs used for removal of the analyses below. (b) JKT00I0 (WT), ROY008 (spt6), JQS002 (WT TATA mutant), and ROY009 (spt6 TATA mutant) previously growing in phosphate containing media (-Pi) were subject to growth in the absence of phosphate (-Pi) to stimulate removal of nucleosomes from the PHO5 promoter, followed by a temperature shift to $39^{\circ} \mathrm{C}$ to inactivate Spt6, followed by addition of phosphate to stimulate removal of the activators from the promoter. Samples at the indicated times after phosphate addition, or in the indicated media were taken for phosphatase activity assays. (c) Samples of strains JKT00I0 (WT), ROY008 (spt6), JQS002 (WT TATA mutant), and ROY009 (spt6 TATA mutant) taken from the same time course in (b) were assessed for histone occupancy over the TATA box of the PHO5 promoter. Data were normalized to the GALI promoter region as a control region, and to the input samples. The average and standard deviation of three independent experiments are plotted. (d) Samples of strains JKT00I0 (WT), ROY008 (spt6), JQS002 (WT TATA mutant), and ROY009 (spt6 TATA mutant) taken from the same time course in (b) were assessed for histone occupancy at the UASp2 binding site of the PHO5 promoter. Data were normalized to the GALI promoter region as a control region, and to the input samples. The average and standard deviation of three independent experiments are plotted. 
tin. Distinction between these two possibilities will be important for understanding the molecular basis of the epigenetic inheritance of a nucleosome-depleted state.

The replication machinery is a highly processive complex that rapidly copies huge numbers of bases while competing with histones, DNA-binding proteins, and transcription factors. Although the replication machinery generally wins out, there are examples of site-specific barrier elements in eukaryotes that mediate replication termination to prevent collisions between the replication machinery and transcription machinery $[28,29]$. Replication forks also pause at stable protein-DNA complexes and within the open reading frames of highly transcribed genes in yeast [30]. However, the PHO5 promoter, along with the rest of the genome, does get replicated and it is hard to imagine how any factor could remain bound to the DNA while the DNA is being threaded through the replication machinery [31]. Indeed the Rrm3 DNA helicase moves with the yeast replication machinery seemingly to remove stably bound proteins from the DNA [32]. Although it has not yet been conclusively addressed for eukaryotic RNA polymerase II transcription, the general transcription machinery is also presumably displaced by passage of the DNA replication machinery. Therefore, following DNA replication, the general transcription machinery reassociates on to the promoter for transcription to proceed and is normally facilitated by transcriptional activators. In our system, the general transcription machinery reassembles on to the nucleosome-depleted $\mathrm{PHO} 5$ promoter in the absence of transcriptional activators in vivo to give efficient transcriptional initiation after DNA replication. As such, activators are not required for transcriptional initiation in vivo if a promoter is nucleosome-depleted, extending our previous observation that activators are not required for transcriptional reinitiation in vivo if a promoter is nucleosome-depleted [13]. Similarly, our work demonstrates that Mediator is not required for transcriptional reinitiation in the absence of activators in vivo at the nucleosome-depleted $\mathrm{PHO} 5$ promoter. This suggests that a critical role of Mediator in vivo is to help activators open up the chromatin structure in order to enable the general transcription machinery to gain access to the core promoter. This idea is consistent with the recently reported role of Mediator in histone $\mathrm{H} 3$ phosphorylation and acetylation [33], revealing a mechanism whereby Mediator helps open up the chromatin structure.

\section{What is the epigenetic mark that specifies a promoter to remain nucleosome-depleted through DNA replication?}

We have ruled out the possibility that transcription per se specifies that the PHO5 promoter should remain nucleosome-depleted, because mutation of the PHO5 TATA box does not prevent inheritance of the nucleosome-depleted promoter through DNA replication. Notably, the TATA box is not required for chromatin disassembly from the PHO5 promoter [34] (Figure 4(c) and 4(d)). It is also possible that a component of the general transcription machinery is bound to the mutant TATA box promoter that prevents chromatin reassembly following DNA replication. Even so, it would be unlikely that such a factor could block chromatin reassembly over both the TATA box region and the adjacent nucleosome to equivalent degrees (as in Figure 4(c) and 4(d)). It is noteworthy that virtually no RNA polymerase II is detected on the mutant TATA box PHO5 promoter [20]. Some activators have been shown to function by a hit-and-run mechanism. For example, the Swi5p activator occupies the HO promoter for only 5 minutes, where it mediates recruitment of SAGA and SWI/SNF, which then allows subsequent recruitment of the activator SBF [35]. However, it is unlikely that Pho4 is acting in this hit-and-run mechanism, as Pho4 is required to occupy continuously the PHO5 promoter to maintain PHO5 transcription, and its dissociation is normally the signal for repression (unless promoter chromatin reassembly is prevented by inactivation of Spt6).

It is also possible that the epigenetic mark for inheritance of nucleosome-depleted DNA is the absence of nucleosomes per se. If chromatin reassembly was directed by the inherited old nucleosomes that are transferred locally to the newly replicated DNA, then the failure to inherit old nucleosomes may be sufficient to maintain the promoter in a nucleosome-depleted state through replication. However, opposing the idea that lack of histones on the parental DNA dictates lack of histones on the newly replicated DNA is the fact that replication-coupled chromatin assembly can be achieved on naked DNA templates in vitro $[36,37]$.

Histone modifications are another way to potentially propagate epigenetic information through DNA replication because the pattern of histone modifications has recently been shown to be preserved through DNA replication [38], when old nucleosomes are distributed randomly on both sides of the fork, with the newly synthesized histones interspersed. In this model, the bromodomains, chromodomains, and so on [39] of chromatin-modifying enzymes would recognize their cognate modifications on the segregated parental histones, permitting the propagation of specific 'histone codes' to adjacent newly assembled nucleosomes following DNA replication [40]. It is possible that histone modifications are involved in the mechanism of epigenetic inheritance of nucleosome-depleted promoters, because the PHO5 promoter is not completely disassembled of chromatin upon transcriptional activation. On average, only three of the four nucleosomes between positions -1 and -4 of the PHO5 promoter are removed from the active promoter 
$[41,42]$. Therefore, it is possible that the nature of the epigenetic mark that specifies a nucleosome-depleted PHO5 promoter may be a specifically modified histone that remains in the -1 to -4 nucleosome positions. Alternatively, the nucleosomes flanking the disassembled region may be specifically modified and signal that the intervening region should remain disassembled. Alternatively, a non-histone factor bound to the PHO5 promoter could signal for chromatin disassembly.

Nucleosomes with dimethylated $\mathrm{K} 36 \mathrm{H} 3$ are refractory to nucleosome disassembly [43], making its depletion an attractive mark for nucleosome disassembly. Consistent with this idea, we find that induction of PHO5 is extremely rapid in yeast deleted for the gene encoding the Set2 methylase for H3 K36 (data not shown). Interestingly, spt6-1004 mutants, but not spt6-140 mutants, lack all H3 K36 methylation presumably due to instability of the Set2 protein $[44,45]$. It will be interesting to investigate in the future whether the histone-depleted $\mathrm{PHO} 5$ promoter is additionally depleted of H3 K36 me2. H3 K36 is a mark for subsequent histone deacetylation $[46,47]$, so its absence would lead to a more acetylated and potentially more readily disassembled promoter. It is also quite likely that a single histone modification will not be sufficient to trigger chromatin disassembly, as these modifications are fairly widespread on the genome. Future studies should reveal the molecular basis for the epigenetic inheritance of the nucleosome-depleted PHO5 promoter, which may serve as a model for understanding the epigenetic inheritance of transcriptional programs in higher eukaryotes that are established by hit-and-run transcriptional activators.

\section{Conclusion}

We have found that the inducibly-nucleosome depleted yeast $\mathrm{PHO} 5$ promoter is not reassembled into chromatin following DNA replication in the absence of the activators that originally signaled for the nucleosome-depletion. As such, this unique system of epigenetic inheritance should facilitate the discovery of the epigenetic mark that maintains the nucleosome-depleted promoter and its transcriptional activity in the absence of activators.

\section{Methods}

\section{Yeast strains and media}

All media used were either YPD (high phosphate) or phosphate-depleted YPD media, made as previously described [48]. Temperature shifts were achieved by spinning down cells and adding prewarmed media to the cell pellet, followed by 4 hours incubation at $39^{\circ} \mathrm{C}$ prior to addition or removal of phosphate. The doubling times for the WT strain at $23^{\circ} \mathrm{C}$ is 120 minutes and for the spt6-140 strain at $23^{\circ} \mathrm{C}$ is 180 minutes. The doubling time for the WT strain at $39^{\circ} \mathrm{C}$ is 190 minutes and for the spt6-140 strain at $39^{\circ} \mathrm{C}$ is 320 minutes. JKT0010, MAT $=$ a his3-11 leu2-3, 112 lys2 trp1-1 ura3-1 bar1::LEU2 w303, is isogenic to ROY008 MAT = a his3-11 leu2-3, 112 lys2 trp1-1 ura3-1 bar1::LEU2 spt6-140 w303. JMY002, which carries the spt6-140 ts mutation has been described previously [13]. MAY0067 carries the spt6-1004 allele and is MAT = a his3-11 leu2-3, 112 lys2 trp1-1 ura3-1 bar1::LEU2 spt6$1004 \mathrm{w} 303$. ROY0010 is MAT $=\mathrm{a}$ ade2 $-1 \operatorname{trp} 1-1$ can $1-100$ leu2-3, 112 his3-11, 15 ura3 GAL+ pho81::TRP1B can1::pPHO5-CAN1 srb4::KANMX6 pRY2844 (LEU2 SRB4+). ROY0011 is MAT $=$ a ade2-1 trp1-1 can1-100 leu2-3, 112 his3-11, 15 ura3 GAL+ pho81::TRP1B can1::pPHO5-CAN1 srb4::KANMX6 pRY2882 (LEU2 srb4-138). The pho80 srb4 double ts mutant was made by transformation of plasmids pRY2844 and pRY2882 and deletion of the endogenous SRB4 locus by deletion cassette replacement, as described previously [49]. Strain SKW066 is derived from strain JMY002, but additionally has the BAR1 gene deleted by insertion of the KanMX6 marker. Strain Z628 carrying the srb4 ts mutant was described previously [49]. Strain KLY042 is derived from strain Z628, but additionally carries SWI1-9myc::URA3 and was described previously [50]. JQS002 carries the PHO5 TATA box mutation and was described previously [20]. ROY009 was derived from JQS002 by insertion of the spt6-140 allele in place of the endogenous SPT6 gene by two-step integration. The spt6 srb4 double ts mutant was generated by dissection of tetrads from diploids that were heterozygous for the two temperature-sensitive mutations. Unless described otherwise, strains were constructed using standard single-step integration methods.

\section{Acid phosphatase activity assays}

Approximately $5 \mathrm{ml}$ of cells were collected by centrifugation and washed with cold $0.1 \mathrm{M}$ sodium acetate $\mathrm{pH}$ 3.6, then resuspended in $500 \mu \mathrm{l}$ of the same buffer. To determine the number of cells used for each reaction, $100 \mu \mathrm{l}$ of the cells were diluted 1:10 in $\mathrm{dd}_{2} \mathrm{O}$ and read at optical density (OD) $600 \mathrm{~nm}$. For each sample reaction, another $100 \mu \mathrm{l}$ of washed cells were diluted 1:5 for a total volume of $500 \mu \mathrm{l}$ in the same sodium acetate buffer and prewarmed for 10 minutes at $30^{\circ} \mathrm{C}$. A $500 \mu \mathrm{l} \mathrm{sample} \mathrm{of} \mathrm{buffer}$ alone was also included as a control, as well as an appropriate volume (500 $\mu \mathrm{l}$ per reaction) of freshly made substrate (nitro phenyl phosphate $0.0742 \mathrm{~g} / 10 \mathrm{ml} 0.1 \mathrm{M}$ sodium acetate $\mathrm{pH}$ 3.6). After warming, $500 \mu \mathrm{l}$ of substrate was added to each reaction sample and incubated at $30^{\circ} \mathrm{C}$ for 10 minutes, at which time $250 \mu \mathrm{l}$ of stop solution, $1 \mathrm{M} \mathrm{Na}_{2} \mathrm{CO}_{3}$, was added. Samples were centrifuged for 1 minute and then read at OD $410 \mathrm{~nm}$. Phosphatase activity was calculated as [(OD $410 \times 1,000) /(O D 600 \times$ volume cell lysate used $(\mu \mathrm{l}) \times$ incubation time (minutes)]. Although single phosphatase time courses are shown in the figures, the results and general trends were all reproduced independently multiple times. 


\section{ChIP analysis}

Yeast cultures $(150 \mathrm{ml})$ were grown to a density of $1 \times 10^{7}$ cells $/ \mathrm{ml}$ and treated with $1 \%$ formaldehyde (final concentration) for 20 minutes at room temperature. Cross-linking was quenched by addition of glycine to a final concentration of $125 \mathrm{mM}$. Cells were sedimented and washed twice in ice-cold Tris-buffered saline $(150 \mathrm{mM}$ $\mathrm{NaCl}, 20 \mathrm{mM}$ Tris $\mathrm{HCl} \mathrm{pH}$ 7.5). Cells were resuspended in $400 \mu \mathrm{l}$ lysis buffer $(0.1 \%$ sodium deoxycholate, $1 \mathrm{mM}$ ethylenediaminetetraacetic acid, $50 \mathrm{mM}$ 4-(2-hydroxyethyl)1-piperazineethanesulfonic acid pH 7.5, $140 \mathrm{mM} \mathrm{NaCl}$, $1 \%$ Triton X-100), an equal volume of $0.5 \mathrm{~mm}$ glass beads were added, and the cells were vortexed for 10 minutes at $4^{\circ} \mathrm{C}$. Chromatin was sheared with a Branson Sonifier 450 to an average size of 500 base pairs. Immunoprecipitations were performed using $2.5 \mu \mathrm{l}$ of the C-terminus antihistone $\mathrm{H} 3$ (Abcam \#ab1791) overnight at $4^{\circ} \mathrm{C}$ as described previously [1]. For Figures 1 and 2, the linear range of template for multiplex polymerase chain reaction (PCR) was determined empirically and PCR-amplified products were quantitatively measured using Labworks (UVP Inc., Upland, CA, USA) as described previously [1].

The ChIP quantitation in Figure 4 was performed by realtime PCR using a Roche Applied Sciences Light Cycler 480. The linear range of PCR templates was determined by performing a 10-fold serial dilution standard curve, which usually proved a 1:10 dilution was sufficient. Each sample was analyzed in triplicate using $10 \mu \mathrm{l}$ reactions in a 384well plate format. The thermal profile was as follows: (1) denaturation at $95^{\circ} \mathrm{C}$ for 10 minutes; (2) run cycle of $95^{\circ} \mathrm{C}$ for 15 seconds then $60^{\circ} \mathrm{C}$ for 1 minute for 50 to 60 cycles; then (3) cooling at $40^{\circ} \mathrm{C}$ for 30 seconds. Each ChIP sample was normalized to its respective Input samples (to account for the number of cells taken), as well as a control region called GAL1/10 whose histone occupancy is regulated by glucose, not phosphate levels.

Primers and Taqman probes used were:

\section{PHO5 UASp2 A: GAATAGGCAATCTCTAAATGAATCGA}

PHO5 UASp2 B: GAAAACAGGGACCAGAATCATAAATT

PHO5 UASp2 probe: FAM-ACCTTGGCACTCACACGTGGGACTAGC-MGB

\section{GAL1/10 A: GACGCACGGAGGAGAGTCTT}

\section{GAL1/10 B: CGCTTAACTGCTCATTGCTATATTG}

GAL1/10 probe: FAM-CGCTCGGCGGCTTCTAATCCGMGB.

\section{Abbreviations}

ChIP: chromatin immunoprecipitation; OD: optical density; PCR: polymerase chain reaction; WT: wild type

\section{Competing interests}

The authors declare that they have no competing interests.

\section{Authors' contributions}

RO and MA performed the experiments. JKT participated in the overall design and coordination of the study and wrote the manuscript. All authors read and approved the final manuscript.

\section{Acknowledgements}

We thank Briana Dennehey and Monica Ransom for careful reading of the manuscript. We are very grateful to Jesper Svejstrup, Rick Young, Mary Ann Osley, Luc Gaudreau, and Fred Winston for the generous gift of strains. The assistance of the University of Colorado Cancer Center Flow Cytometry Core Facility was invaluable for this study. This work was supported by a Mochida Memorial Foundation for Medical and Pharmaceutical Research Travel Award, a University of Colorado Cancer Center Fellowship and a Cancer League of Colorado Fellowship to RO and an NIH ROI grant GM64475 to JKT.

\section{References}

I. Adkins MW, Howar SR, Tyler JK: Chromatin disassembly mediated by the histone chaperone Asfl is essential for transcriptional activation of the yeast $\mathrm{PHO} 5$ and $\mathrm{PHO}$ genes. Mol Cell 2004, I4(5):657-666.

2. Boeger H, Griesenbeck J, Strattan JS, Kornberg RD: Removal of promoter nucleosomes by disassembly rather than sliding in vivo. Mol Cell 2004, I 4(5):667-673.

3. Lee CK, Shibata Y, Rao B, Strahl BD, Lieb JD: Evidence for nucleosome depletion at active regulatory regions genome-wide. Nat Genet 2004, 36(8):900-905.

4. Bernstein BE, Liu CL, Humphrey EL, Perlstein EO, Schreiber SL: Global nucleosome occupancy in yeast. Genome Biol 2004, 5(9):R62.

5. Chen X, Wang J, Woltring D, Gerondakis S, Shannon MF: Histone dynamics on the interleukin-2 gene in response to $\mathrm{T}$-cell activation. Mol Cell Biol 2005, 25(8):3209-3219.

6. Schwabish MA, Struhl K: Evidence for eviction and rapid deposition of histones upon transcriptional elongation by RNA polymerase II. Mol Cell Biol 2004, 24(23): I0III-10II7.

7. Choder M, Bratosin S, Aloni $\mathrm{Y}$ : A direct analysis of transcribed minichromosomes: all transcribed SV40 minichromosomes have a nuclease-hypersensitive region within a nucleosomefree domain. Embo ] 1984, 3( I 2):2929-2936.

8. Mito Y, Henikoff JG, Henikoff S: Genome-scale profiling of histone H3.3 replacement patterns. Nat Genet 2005, 37(10): 1090-1097.

9. Zhao J, Herrera-Diaz J, Gross DS: Domain-wide displacement of histones by activated heat shock factor occurs independently of Swi/Snf and is not correlated with RNA polymerase II density. Mol Cell Biol 2005, 25(20):8985-8999.

10. Herrera F], Triezenberg SJ: VP I6-dependent association of chromatin-modifying coactivators and underrepresentation of histones at immediate-early gene promoters during herpes simplex virus infection. J Virol 2004, 78( I 8):9689-9696.

II. Pokholok DK, Harbison CT, Levine S, Cole M, Hannett NM, Lee TI, Bell GW, Walker K, Rolfe PA, Herbolsheimer E, et al:: Genomewide map of nucleosome acetylation and methylation in yeast. Cell 2005, I 22(4):517-527.

12. Adkins MW, Williams SK, Linger J, Tyler JK: Chromatin disassembly from the PHO5 promoter is essential for the recruitment of the general transcription machinery and coactivators. Mol Cell Biol 2007, 27( I 8):6372-6382. 
13. Adkins MW, Tyler JK: Transcriptional activators are dispensable for transcription in the absence of Spt6-mediated chromatin reassembly of promoter regions. Mol Cell 2006, 2I:405-4I6.

14. O'Neill EM, Kaffman A, Jolly ER, O'Shea EK: Regulation of PHO4 nuclear localization by the PHO80-PHO85 cyclin-CDK complex. Science 1996, 27I(5246):209-2I2.

15. Barbaric S, Munsterkotter M, Svaren J, Horz W: The homeodomain protein $\mathrm{Pho2}$ and the basic-helix-loop-helix protein Pho4 bind DNA cooperatively at the yeast PHO5 promoter. Nucleic Acids Res 1996, 24(22):4479-4486.

16. Svaren J, Horz W: Interplay between nucleosomes and transcription factors at the yeast PHO5 promoter. Semin Cell Biol 1995, 6(4): 177-183.

17. Kaffman A, Herskowitz I, Tjian R, O'Shea EK: Phosphorylation of the transcription factor PHO4 by a cyclin-CDK complex, PHO80-PHO85. Science 1994, 263(5 I 50): II53-II 56.

18. Reeves WM, Hahn S: Activator-independent functions of the yeast mediator $\sin 4$ complex in preinitiation complex formation and transcription reinitiation. Mol Cell Biol 2003 23(I):349-358.

19. Baek HJ, Kang YK, Roeder RG: Human Mediator enhances basal transcription by facilitating recruitment of transcription factor IIB during preinitiation complex assembly. J Biol Chem 2006, 28I(22): $|5| 72-1518 \mid$.

20. Uhler JP, Hertel C, Svejstrup JQ: A role for noncoding transcription in activation of the yeast PHO5 gene. Proc Natl Acad Sci USA 2007, 104(19):8011-8016.

21. Kaffman A, Rank NM, O'Neill EM, Huang LS, O'Shea EK: The receptor Msn5 exports the phosphorylated transcription factor Pho4 out of the nucleus. Nature 1998, 396(67 I0):482-486.

22. Springer M, Wykoff DD, Miller N, O'Shea EK: Partially Phosphorylated Pho4 Activates Transcription of a Subset of Phosphate-Responsive Genes. PLoS Biol 2003, I(2):E28.

23. Komeili A, O'Shea EK: Roles of phosphorylation sites in regulating activity of the transcription factor Pho4. Science 1999, 284(54 I6): $977-980$

24. Yuan GC, Liu YJ, Dion MF, Slack MD, Wu LF, Altschuler SJ, Rando OJ: Genome-scale identification of nucleosome positions in $\mathbf{S}$. cerevisiae. Science 2005, 309(5734):626-630.

25. Struhl K: Naturally occurring poly(dA-dT) sequences are upstream promoter elements for constitutive transcription in yeast. Proc Natl Acad Sci USA 1985, 82(24):8419-8423.

26. Anderson JD, Widom J: Poly(dA-dT) promoter elements increase the equilibrium accessibility of nucleosomal DNA target sites. Mol Cell Biol 200I, 2I(I I):3830-3839.

27. Nelson HC, Finch JT, Luisi BF, Klug $A$ : The structure of an oligo(dA).oligo(dT) tract and its biological implications. Nature 1987, 330(6 I 45):22I-226.

28. Dalgaard JZ, Klar AJ: A DNA replication-arrest site RTSI regulates imprinting by determining the direction of replication at mat I in S. pombe. Genes Dev 200I, I 5(16):2060-2068.

29. Brewer BJ, Fangman WL: A replication fork barrier at the 3 ' end of yeast ribosomal RNA genes. Cell 1988, 55(4):637-643.

30. Azvolinsky A, Giresi PG, Lieb JD, Zakian VA: Highly transcribed RNA polymerase II genes are impediments to replication fork progression in Saccharomyces cerevisiae. Mol Cell 2009, 34(6):722-734.

31. Klinge S, Nunez-Ramirez R, Llorca O, Pellegrini L: 3D architecture of DNA Pol alpha reveals the functional core of multi-subunit replicative polymerases. EMBO / 2009, 28( I3): 1978- I 987.

32. Azvolinsky A, Dunaway S, Torres JZ, Bessler JB, Zakian VA: The S. cerevisiae Rrm3p DNA helicase moves with the replication fork and affects replication of all yeast chromosomes. Genes Dev 2006, 20(22):3104-3116.

33. Meyer KD, Donner AJ, Knuesel MT, York AG, Espinosa JM, Taatjes D): Cooperative activity of cdk8 and GCN5L within Mediator directs tandem phosphoacetylation of histone $\mathbf{H} 3$. EMBO J 2008, 27(10): 1447-1457.

34. Fascher KD, Schmitz J, Horz W: Structural and functional requirements for the chromatin transition at the $\mathrm{PHO} 5$ promoter in Saccharomyces cerevisiae upon PHO5 activation. J Mol Biol I993, 23I(3):658-667.

35. Cosma MP, Tanaka T, Nasmyth K: Ordered recruitment of transcription and chromatin remodeling factors to a cell cycle- and developmentally regulated promoter. Cell 1999 , 97(3):299-3II.

36. Smith S, Stillman B: Purification and characterization of CAF-I, a human cell factor required for chromatin assembly during DNA replication in vitro. Cell 1989, 58(I):15-25.

37. Tyler JK, Adams CR, Chen SR, Kobayashi R, Kamakaka RT, Kadonaga JT: The RCAF complex mediates chromatin assembly during DNA replication and repair. Nature 1999, 402(6761):555-560.

38. Benson LJ, Gu Y, Yakovleva T, Tong K, Barrows C, Strack CL, Cook RG, Mizzen CA, Annunziato AT: Modifications of H3 And H4 during chromatin replication, nucleosome assembly, and histone exchange. J Biol Chem 2006, 28 I ( I 4):9287-9296.

39. de la Cruz X, Lois S, Sanchez-Molina S, Martinez-Balbas MA: Do protein motifs read the histone code? Bioessays 2005, 27(2): I64- 175.

40. Felsenfeld G, Groudine M: Controlling the double helix. Nature 2003, 42 I (692I):448-453.

4I. Boeger H, Griesenbeck J, Strattan JS, Kornberg RD: Nucleosomes unfold completely at a transcriptionally active promoter. Mol Cell 2003, I I(6): I587-I598.

42. Bergman LW, Stranathan MC, Preis LH: Structure of the transcriptionally repressed phosphate-repressible acid phosphatase gene (PHO5) of Saccharomyces cerevisiae. Mol Cell Biol 1986, 6(1):38-46

43. Rao B, Shibata Y, Strahl BD, Lieb JD: Dimethylation of histone $\mathbf{H 3}$ at lysine 36 demarcates regulatory and nonregulatory chromatin genome-wide. Mol Cell Biol 2005, 25(2 I):9447-9459.

44. Chu Y, Sutton A, Sternglanz R, Prelich G: The BURI cyclindependent protein kinase is required for the normal pattern of histone methylation by SET2. Mol Cell Biol 2006, 26(8):3029-3038.

45. Youdell ML, Kizer KO, Kisseleva-Romanova E, Fuchs SM, Duro E, Strahl BD, Mellor J: Roles for Ctk I and Spt6 in regulating the different methylation states of histone $\mathrm{H} 3$ lysine 36. Mol Cell Biol 2008, 28( I6):4915-4926.

46. Carrozza MJ, Li B, Florens L, Suganuma T, Swanson SK, Lee KK, Shia W], Anderson S, Yates J, Washburn MP, et al.: Histone $\mathbf{H 3}$ methylation by Set2 directs deacetylation of coding regions by Rpd3S to suppress spurious intragenic transcription. Cell 2005, I 23(4):58I-592.

47. Keogh MC, Kurdistani SK, Morris SA, Ahn SH, Podolny V, Collins SR, Schuldiner M, Chin K, Punna T, Thompson NJ, et al.: Cotranscriptional set 2 methylation of histone $\mathrm{H} 3$ lysine 36 recruits a repressive Rpd3 complex. Cell 2005, I 23(4):593-605.

48. Han M, Kim UJ, Kayne P, Grunstein M: Depletion of histone H4 and nucleosomes activates the PHO5 gene in Saccharomyces cerevisiae. Embo J 1988, 7(7):2221-2228.

49. Thompson CM, Young RA: General requirement for RNA polymerase II holoenzymes in vivo. Proc Natl Acad Sci USA 1995, 92(10):4587-4590.

50. Lemieux K, Gaudreau L: Targeting of Swi/Snf to the yeast GALI UAS G requires the Mediator, TAF IIs, and RNA polymerase II. EMBO / 2004, 23(20):4040-4050.

Publish with Bio Med Central and every scientist can read your work free of charge

"BioMed Central will be the most significant development for disseminating the results of biomedical research in our lifetime. "

Sir Paul Nurse, Cancer Research UK

Your research papers will be:

- available free of charge to the entire biomedical community

- peer reviewed and published immediately upon acceptance

- cited in PubMed and archived on PubMed Central

- yours - you keep the copyright
BioMedcentral 